\title{
Soft-sensing and Error Analysis of Air Flow
}

\author{
LI Jian ", ZHANG Bin-wen \\ North China Electric Power University, No.2 Beinong Road, Changping District, Beijing, China \\ xlij8802@163.com
}

Keywords: air flow; soft-sensing; error analysis; data fusion

\begin{abstract}
To measure air flow in the power plant, regression models are set up with variables according to mechanical analysis and data mining technique, and the results are verified by goodness of fit, F-test and deviance. It is proved that the results using multi-sensor data fusion is more reliable and accurate than the single ones or the arithmetic average value, and based on that, a principle is presented to ensure the data with smaller error is selected. The soft-sensing model of air flow is established by the experiment in a power plant.
\end{abstract}

\section{Introduction}

The main air measuring points in the power plant boiler include the outlet hot primary and secondary and the cold primary air flow of the air preheater, the mixed cold airflow in the mill's entrance. There are mainly two methods for the air flow measurement: according to differential pressure and heat diffusion. The flowmeters of differential pressure type calculate the flow via measuring the differential pressure of the throttling element based on the Bernouli Equation and Continuity Equation of Fluid. There are errors due to its non standard measuring device, the flow coefficient changes influenced by the flow field, the limitation of installation, fly ash and fluctuations, etc. The ones of heat diffusion type calculate the flow by measuring the temperature change of two thermal resistances, without pressure loss and temperature compensation. They are stable with small pressure loss and no blockage, but lag because of the process of heating and cooling of the thermal resistances and easily affected by the pulsating flow [1-4].

According to the problems, on the one hand, engineers upgrade the existing devices to reduce errors, such as using double Venturi tubes to increase the pressure signal, improving the type to reduce the pressure loss and straight pipe section and cleaning automatically. On the other hand, researchers found that the air flow can be calculated through the performance monitoring and soft-sensing using some auxiliary variables [5-6]. In recent years, the soft measurement technology has been widely used in the research of thermal power plant.

\section{Soft-sensing Modeling}

The application of the air flow soft measurement methods include artificial intelligence techniques and fitting method based on the performance curve and operation data, having different calculation, fitting accuracy and applicable scope.

Auxiliary Variables. The selection of secondary variables is critical for the soft measurement results. The auxiliary variables must first have a close relationship with the measured variable and every single one should be easily measured and have high accuracy. The number and position of the auxiliary variables are also important.

A soft measurement model was proposed in [4] based on the theory of coal combustion applies coal quality, coal quantity and oxygen content in the flue gas to calculate the total amount of air. The calculation formula is

$$
V_{a}=\frac{0.266 K_{c 1} Q_{B}\left(\omega\left(O_{2}\right)^{\prime}\left(K_{c 2}-1\right)+\omega_{O_{2}}\right)}{\omega_{O_{2}}-\omega\left(O_{2}\right)^{\prime}}
$$


where $K_{c 1}$ and $K_{c 2}$ are coal quality coefficient, $\omega\left(O_{2}\right)^{\prime}$ is oxygen content in dry flue gas, $\omega_{O_{2}}$ is oxygen content in dry air, and $Q_{B}$ is total heat generated by coal.

The method of performance curve in [5] treats the fan as a part of pressure, so the parameters can be attributed to the flow, pressure, temperature and density. By the way the changes of temperature and density can be ignored with the assumption that the pressure and power are not affected by the effects of transient phenomena. Thus the flow model can be achieved by the total pressure of the fan, deflector opening and power coefficient.

It is found in [6] that there is a strong correlation among the motor current, blower opening, pressure difference and air volume.

It gives the calculation of current and power in [7] as:

$$
\begin{aligned}
& I=\frac{N \cdot 1000}{U \cdot \cos \varphi \cdot \eta_{e} \cdot \sqrt{3}} \mathrm{~A} \\
& N=\frac{Q \cdot p}{3600 \cdot 1000 \cdot \eta} \mathrm{kW}
\end{aligned}
$$

where $U$ is the voltage [V], $\cos \varphi$ is the motor power coefficient, $\eta_{e}$ is the motor efficient, $Q$ is the flow $\left[\mathrm{m}^{3} / \mathrm{h}\right], p$ is the total pressure $[\mathrm{Pa}]$, and $\eta$ is the air efficient [\%].

Model Test. We always test the validation of models after they are established. The goodness of fit is calculated by the correlation coefficient between the measurement and the estimate

$$
r=\frac{\sum_{i=1}^{N}\left(y_{i}-\bar{y}\right)\left(\hat{y}_{i}-\overline{\hat{y}}\right)}{\sqrt{\sum_{i=1}^{N}\left(y_{i}-\bar{y}\right)^{2}} \sqrt{\sum_{i=1}^{N}\left(\hat{y}_{i}-\overline{\hat{y}}\right)^{2}}}
$$

Significance test uses the $F$ statistical value which can be written as

$$
F=\frac{\sum_{i=1}^{N}\left(\hat{y}_{i}-\bar{y}\right)^{2} / M}{\sum_{i=1}^{N}\left(y_{i}-\hat{y}_{i}\right)^{2} /(N-M-1)}
$$

where $M$ is the number of auxiliary variables.

Residual variance is calculated as follows

$$
\sigma^{2}=\frac{\sum_{i=1}^{N}\left(y_{i}-\hat{y}_{i}\right)^{2}}{N-M-1}
$$

Data Fusion. When there are a few measuring sensors or models, we can combine them using weighted fusion algorithm to obtain a more reliable result.

$$
Y=\alpha_{1} Y_{1}+\alpha_{2} Y_{2}+\mathrm{L}+\alpha_{n} Y_{n}
$$

where the weight is defined as 


$$
\alpha_{i}=\frac{1 / \sigma_{i}^{2}}{\sum_{i=1}^{n} 1 / \sigma_{i}^{2}}
$$

and the variance is given by

$$
\sigma=\sqrt{\sum_{i=1}^{n}\left(\frac{\partial Y}{\partial Y_{i}}\right)^{2} \sigma_{i}^{2}}=\frac{1}{\sqrt{\sum_{i=1}^{n} 1 / \sigma_{i}^{2}}}
$$

As shown above, the more measurements we have the higher accuracy the fusion result has. However, if the measurement's weight is quite small while its variance is big, it is unnecessary to put it in the fusion. A measurement is selected which satisfies the following criteria:

$$
\sigma_{i} \leq t \sigma_{\min }
$$

where $t$ is a confidence coefficient determined by error distribution.

\section{Illustrative Case}

Data from a 1000MW power plant in China is used, whose boiler type is SG3040-27.56-M538 and fan blower is ANN-3120/1600N.

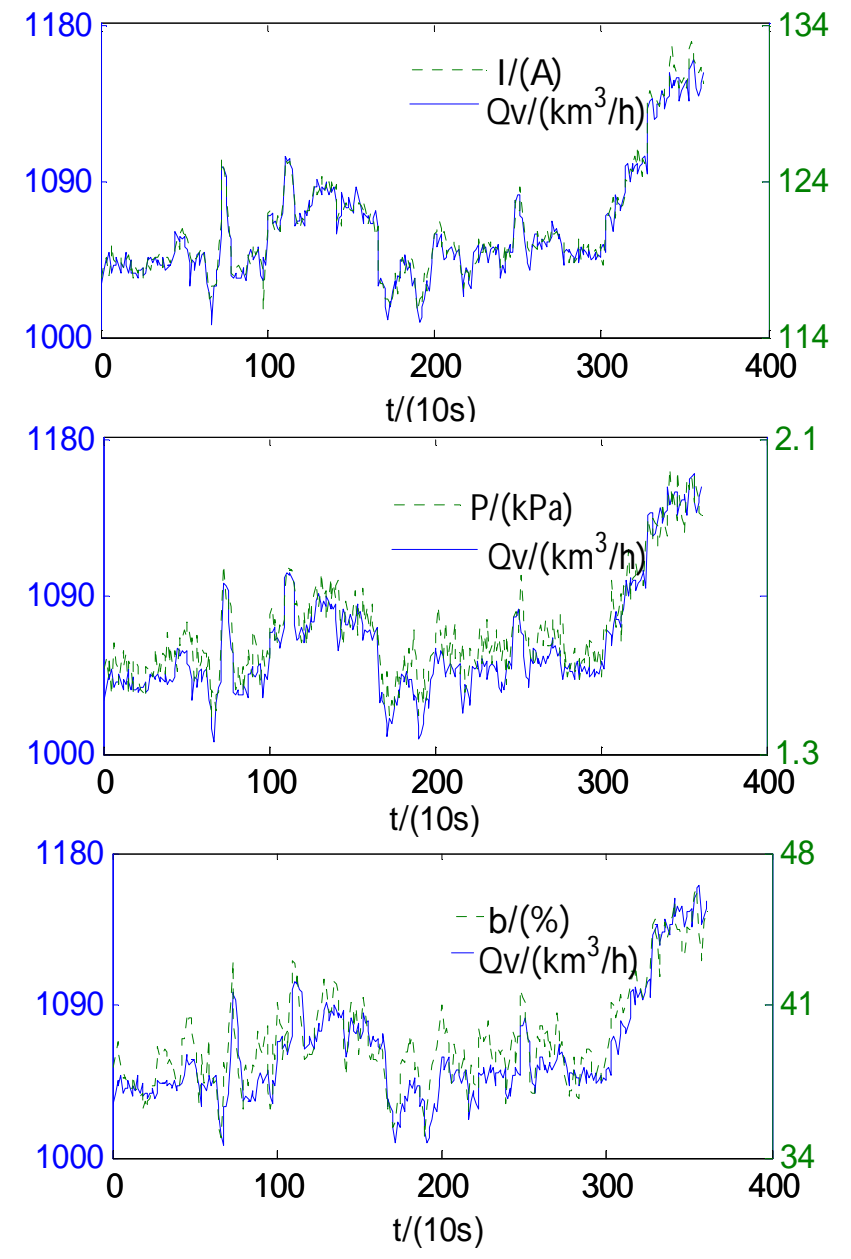

Fig. 1 The Comparison between $I, P, \beta$ and $Q_{v}$ 
The trend chart (Fig. 1) shows flow changes as current $I$, pressure $P$ and opening $\beta$, so these three variables are selected as inputs of soft-sensing models.

We use the auxiliary variables to establish single equation econometric models of airflow which can be shown as:

Table 1 Model Test Result

\begin{tabular}{cccc}
\hline Model & Goodness of Fit & F & Standard Deviation \\
\hline$\hat{Q}_{v 1}=8.6176 I+18.5526$ & 0.9791 & 8317.38 & 6.5547 \\
$\hat{Q}_{v 2}=12.7394 \beta+560.035$ & 0.9119 & 1772.30 & 13.2251 \\
$\hat{Q}_{v 3}=230.4319 P+690.7904$ & 0.9230 & 2064.73 & 12.4016 \\
\hline
\end{tabular}

All three models pass the goodness of fit and $\mathrm{F}$ test. The minimum standart deviation of them is 6.5547, and its confidence coefficient can be calculated as 1.96 . So the model $\hat{Q}_{v 1}$ and $\hat{Q}_{v 3}$ are proposed to put in the fusion process while they satisfy Eq. 10.

\section{Summary}

Soft-sensing models for air flow in power plant has been designed, established and evaluated, which can be an effective reference. It can work instead for a short period while the measuring devices are in the fault diagnosis because of its accuracy and response speed. The fusion theory proves that it has a more accurate result than any original sensor and arithmetic mean value of them, and a selecting principle has been presented to ensure that the sensors with smaller error will be put in.

\section{References}

[1] CHENG Qi-ming, CHENG Yin-man, WANG Ming-mei, WANG Ying-fei, Current Situation and Development of the Measuring Techniques for Primary and Secondary Air Speed in Boiler Control of Fossil-fired Power Plant, Process Automation Instrumentation 32 (7) (2011)

[2] ZHANG Bo, HAO Zhen-hua, Interlock and Application of Boiler PA and SA System Air Flow Measuring Devices, Power System Engineering 28 (4) (2012) 56-57

[3] WANG Xu-bo, LI Guang-hong, WANG Wei, Thermal power plant directly firing pulverized coal boiler coal mill once wind measurement and automatic control optimization, Journal of Shaanxi University of Technology (Natural Science Edition) 29 (1) (2013) 5-11

[4] TIAN Liang, HUO Qiu-bao, LIU Xin-ping, LIU Ji-zhen, Soft-sensors of the Total Air Volume in Utility Boilers, Proceedings of the CSEE 34 (8) (2014) 1261-1267

[5] HOU Jun-hu, WANG Song-ling, AN Lian-suo, HU Hai-yan, Experimental Study on Flow Measurement of Fan Based on Parameter Mapping, Proceedings of the CSEE 23 (10) (2003) 209-214

[6] LIU Ji-zhen, ZHAO Zheng, LIU Jin-kang, ZENG De-liang, Soft-sensing models of air flow based on data fusion, Journal of North China Electric Power University 32 (3) (2005) 61-65

[7] WU Bi-ke, Cause Analysis and Countermeasures of Large Deviation of IDF Current in Utility Boiler, Power System Engineering 20 (5) (2004) 11-14

(Supported by "the Fundamental Research Funds for the Central Universities” 2015MS26) 